\title{
Exact Random Coding Exponents for Erasure Decoding
}

\author{
Anelia Somekh Baruch \\ School of Engineering \\ Bar-Ilan University \\ Ramat-Gan, Israel 52900 \\ Email: anelia.somekhbaruch@gmail.com
}

\author{
Neri Merhav \\ Department of Electrical Engineering \\ Technion Israel Institute of Technology \\ Haifa, Israel 32000 \\ Email: merhav@ee.technion.ac.il
}

\begin{abstract}
Random coding of a channel with an erasure option is studied. By analyzing the large deviations behavior of the code ensemble, we obtain exact single-letter formulas for the error exponents in lieu of Forney's lower bounds. The analysis technique we use is based on an enhancement and specialization of tools for assessing the moments of certain distance enumerators, that were recently used for determining the exponential behavior of other communication systems. We specialize our results to the binary symmetric channel case with uniform random coding distribution and derive an explicit expression for the error exponent which, unlike Forney's bounds, does not involve optimization over two parameters. We also establish the fact that for the binary symmetric channel case with uniform random coding distribution, the difference between the exact error exponent corresponding to the probability of undetected decoding error and the error exponent corresponding to the erasure event is equal to the threshold parameter. Numerical calculations for the binary symmetric channel with uniform random coding distribution indicate that in this case Forney's bound coincides with the exact random coding exponent.
\end{abstract}

\section{INTRODUCTION}

In [1], Forney derived lower bounds on the random coding exponents associated with decoding rules that allow for erasure and list decoding (see also later related studies [2] - [7]). The channel model he considered was a single user discrete memoryless channel (DMC) where a codebook of block length $n$ is randomly drawn with i.i.d. codewords having i.i.d. symbols. When erasure is concerned, the decoder may fully decode the message, or, decide to declare that an erasure has occurred. An optimum tradeoff between the probability of erasure and the probability of undetected decoding error was investigated. This tradeoff is optimally controlled by a threshold parameter $T$ of the function $e^{n T}$ to which one compares the ratio between the likelihood of each hypothesized message and the sum of likelihoods of all other messages. If this ratio exceeds $e^{n T}$ for some message, a decision is made in favor of that message, otherwise, an erasure is declared. Forney's main result in [1] is a single-letter lower bound $E_{1}(R, T)$ to the exponent of the probability of the event $\mathcal{E}_{1}$ of not making the correct decision, namely, either erasing or making the wrong decision, and a single letter lower bound $E_{2}(R, T)$ to the exponent of the probability of the event $\mathcal{E}_{2}$ of undetected error.

Inspired by statistical a mechanical point of view on random code ensembles (offered in [8] and further elaborated on in
[11]), Merhav [9] applied a different technique to derive a lower bound to the exponents of the probabilities of $\mathcal{E}_{1}, \mathcal{E}_{2}$ by assessing the moments of certain distance enumerators. This approach, which proved fruitful in many other applications (see [10], [12], [13]) resulted in a bound that is at least as tight as Forney's bound. It is shown in [9] that under certain symmetry conditions (that often hold) on the random coding distribution and the channel, the resulting bound is also simpler in the sense that there is only one parameter to optimize rather than two. Moreover, this optimization can be carried out in closed form at least in some special cases like the binary symmetric channel (BSC). It is not clear though, whether the bounds of [9] are strictly tighter than those of Forney or not.

In this paper, we use the approach of distance enumerators to tackle again the problem of random coding error exponents of channel with an erasure option. Unlike the approach of [1] and [9], our starting point is not a Gallager-type bound [14] on the probability of error, but rather analyzing the exact expression. This approach results in single-letter expressions for the exact exponential behavior of the probabilities of the events $\mathcal{E}_{1}, \mathcal{E}_{2}$ when random coding is used. So far, we have not been able to determine analytically whether our results coincide with Forney's bounds, i.e., we cannot say whether Forney's bounds are tight or not, but the tightness of the expressions we obtain is guaranteed.

The outline of this paper is as follows. In Section II, we present notation conventions and in Section III, we give some necessary background in more detail. Section IV is devoted to description of the main results. We conclude In Section V, where we derive the exact expression for the error exponent corresponding to the probability of $\mathcal{E}_{1}$.

\section{NOTATION}

Throughout this paper, scalar random variables (RVs) will be denoted by capital letters, their sample values will be denoted by the respective lower case letters, and their alphabets will be denoted by the respective calligraphic letters, e.g. $X$, $x$, and $\mathcal{X}$, respectively. A similar convention will apply to random vectors of dimension $n$ and their sample values, which will be denoted with same symbols in the bold face font. The set of all $n$-vectors with components taking values in a certain finite alphabet, will be denoted as the same alphabet 
superscripted by $n$, e.g., $\mathcal{X}^{n}$. Sources and channels will be denoted generically by the letter $P$ or $Q$. Information theoretic quantities, such as entropies and conditional entropies, will be denoted following the usual conventions of the information theory literature, e.g., $H(X), H(X \mid Y)$, and so on. When we wish to emphasize the dependence of the entropy on a certain underlying probability distribution, say $Q$, we subscript it by $Q$, i.e., use notations like $H_{Q}(X), H_{Q}(X \mid Y)$, etc. The expectation operator will be denoted by $\boldsymbol{E}\{\cdot\}$, and once again, when we wish to make the dependence on the underlying distribution $Q$ clear, we denote it by $\boldsymbol{E}_{Q}\{\cdot\}$. The cardinality of a finite set $A$ will be denoted by $|A|$. The indicator function of an event $\mathcal{E}$ will be denoted by $1\{\mathcal{E}\}$. For a given sequence $\boldsymbol{y} \in \mathcal{Y}^{n}, \mathcal{Y}$ being a finite alphabet, $\hat{P}_{\boldsymbol{y}}$ will denote the empirical distribution on $\mathcal{Y}$ extracted from $\boldsymbol{y}$, in other words, $\hat{P} \boldsymbol{y}$ is the vector $\left\{\hat{P}_{\boldsymbol{y}}(y), y \in \mathcal{Y}\right\}$, where $\hat{P}_{\boldsymbol{y}}(y)$ is the relative frequency of the letter $y$ in the vector $\boldsymbol{y}$. For two sequences of positive numbers, $\left\{a_{n}\right\}$ and $\left\{b_{n}\right\}$, the notation $a_{n} \doteq b_{n}$ means that $\left\{a_{n}\right\}$ and $\left\{b_{n}\right\}$ are of the same exponential order, i.e., $\frac{1}{n} \ln \frac{a_{n}}{b_{n}} \rightarrow 0$ as $n \rightarrow \infty$. Similarly, $a_{n} \leq b_{n}$ means that $\limsup \sup _{n} \frac{1}{n} \ln \frac{a_{n}}{b_{n}} \leq 0$, and so on. Another notation that we shall use is that for a real number $x,|x|^{+}=\max \{0, x\}$.

\section{Preliminaries}

Consider a DMC with a finite input alphabet $\mathcal{X}$, finite output alphabet $\mathcal{Y}$, and single-letter transition probabilities $\{P(y \mid x), x \in \mathcal{X}, y \in \mathcal{Y}\}$. As the channel is fed by an input vector $\boldsymbol{x} \in \mathcal{X}^{n}$, it generates an output vector $\boldsymbol{y} \in \mathcal{Y}^{n}$ according to the sequence of conditional probability distributions $P\left(y_{i} \mid x_{1}, \ldots, x_{i}, y_{1}, \ldots, y_{i-1}\right)=P\left(y_{i} \mid x_{i}\right), i=1,2, \ldots, n$ where for $i=1,\left(y_{1}, \ldots, y_{i-1}\right)$ is understood as the null string. A rate$R$ block code of length $n$ consists of $M=e^{n R} n$-vectors $\boldsymbol{x}_{m}$, $m=1,2, \ldots, M$, which represent $M$ different messages. We will assume that all possible messages are a-priori equiprobable, i.e., $P(m)=1 / M$ for all $m=1,2, \ldots, M$. A decoder with an erasure option is a partition of $\mathcal{Y}^{n}$ into $(M+1)$ regions, $\mathcal{R}_{0}, \mathcal{R}_{1}, \ldots, \mathcal{R}_{M}$. Such a decoder works as follows: If $\boldsymbol{y}$ falls into $R_{m}, m=1,2, \ldots, M$, then a decision is made in favor of message number $m$. If $\boldsymbol{y} \in \mathcal{R}_{0}$, no decision is made and an erasure is declared. We will refer to $\mathcal{R}_{0}$ as the erasure event. Given a code $C=\left\{\boldsymbol{x}_{1}, \ldots, \boldsymbol{x}_{M}\right\}$ and a decoder $\mathcal{R}=\left(\mathcal{R}_{0}, \mathcal{R}_{1}, \ldots, R_{m}\right)$, let us now define two undesired events. The event $\mathcal{E}_{1}$ is the event of not making the right decision. This event is the disjoint union of the erasure event and the event $\mathcal{E}_{2}$, which is the undetected error event, namely, the event of making the wrong decision. The probabilities of all three events are defined as follows:

$$
\begin{aligned}
& \operatorname{Pr}\left\{\mathcal{E}_{1}\right\}=\sum_{m=1}^{M} \sum_{\boldsymbol{y} \in \mathcal{R}_{m}^{c}} P\left(\boldsymbol{x}_{m}, \boldsymbol{y}\right)=\frac{1}{M} \sum_{m=1}^{M} \sum_{\boldsymbol{y} \in \mathcal{R}_{m}^{c}} P\left(\boldsymbol{y} \mid \boldsymbol{x}_{m}\right) \\
& \operatorname{Pr}\left\{\mathcal{E}_{2}\right\}=\frac{1}{M} \sum_{m=1}^{M} \sum_{\boldsymbol{y} \in \mathcal{R}_{m}} \sum_{m^{\prime} \neq m} P\left(\boldsymbol{y} \mid \boldsymbol{x}_{m^{\prime}}\right)
\end{aligned}
$$

$$
\operatorname{Pr}\left\{\mathcal{R}_{0}\right\}=\operatorname{Pr}\left\{\mathcal{E}_{1}\right\}-\operatorname{Pr}\left\{\mathcal{E}_{2}\right\} .
$$

Forney [1] shows, using the Neyman-Pearson Theorem, that the best tradeoff between $\operatorname{Pr}\left\{\mathcal{E}_{1}\right\}$ and $\operatorname{Pr}\left\{\mathcal{E}_{2}\right\}$ is attained by the decoder $\mathcal{R}^{*}=\left(\mathcal{R}_{0}^{*}, \mathcal{R}_{1}^{*}, \ldots, \mathcal{R}_{M}^{*}\right)$ defined by

$$
\begin{aligned}
& \mathcal{R}_{m}^{*}=\left\{\boldsymbol{y}: \frac{P\left(\boldsymbol{y} \mid \boldsymbol{x}_{m}\right)}{\sum_{m^{\prime} \neq m} P\left(\boldsymbol{y} \mid \boldsymbol{x}_{m^{\prime}}\right)} \geq e^{n T}\right\}, m=1,2, \ldots, M \\
& \mathcal{R}_{0}^{*}=\bigcup_{m=1}^{M}\left(\mathcal{R}_{m}^{*}\right)^{c},
\end{aligned}
$$

where $\left(\mathcal{R}_{m}^{*}\right)^{c}$ is the complement of $\mathcal{R}_{m}^{*}$, and where $T \geq 0$ is a parameter, henceforth referred to as the threshold, which controls the balance between the probabilities of $\mathcal{E}_{1}$ and $\mathcal{E}_{2}$.

Define the error exponents $e_{i}(R, T), i=1,2$, as the exponents associated with the average probabilities of error $\overline{\operatorname{Pr}}\left\{\mathcal{E}_{i}\right\}, i=1,2$ where the average is taken with respect to (w.r.t.) the ensemble of randomly selected codes, drawn independently according to an i.i.d. distribution $P(\boldsymbol{x})=$ $\prod_{i=1}^{n} P\left(x_{i}\right)$, that is,

$$
e_{i}(R, T)=\underset{n}{\limsup }\left[-\frac{1}{n} \ln \overline{\operatorname{Pr}}\left\{\mathcal{E}_{i}\right\}\right], \quad i=1,2 .
$$

Forney derives lower bounds, $E_{1}(R, T)$ and $E_{2}(R, T)$, to $e_{1}(R, T)$ and $e_{2}(R, T)$, (or, upper bounds to the average probabilities of error), respectively, given by

$$
E_{1}(R, T)=\max _{0 \leq s \leq \rho \leq 1}\left[E_{0}(s, \rho)-\rho R-s T\right],
$$

where

$E_{0}(s, \rho)=$

$-\ln \left[\sum_{y}\left(\sum_{x} P(x) P^{1-s}(y \mid x)\right)\left(\sum_{x^{\prime}} P\left(x^{\prime}\right) P^{s / \rho}\left(y \mid x^{\prime}\right)\right)^{\rho}\right]$

and

$$
E_{2}(R, T)=E_{1}(R, T)+T .
$$

Merhav [9] established tighter (though not necessarily strictly tighter) upper bounds to $e_{1}(R, T)$ and $e_{2}(R, T)$ denoted $E_{1}^{*}(R, T)$ and $E_{2}^{*}(R, T)$. These bounds take on a very simple form under the following condition:

Condition 1 The random coding distribution $\{P(x), x \in \mathcal{X}\}$ and the channel transition matrix $\{P(y \mid x), x \in \mathcal{X}, y \in \mathcal{Y}\}$ are such that for every real $s$,

$$
\gamma_{y}(s) \triangleq-\ln \left[\sum_{x \in \mathcal{X}} P(x) P^{s}(y \mid x)\right]
$$

is independent of $y$, in which case, it will be denoted by $\gamma(s)$.

Under Condition 1, the bounds to the exponents are

$$
E_{1}^{*}(R, T)=\sup _{s \geq 0}[\Lambda(R, s)+\gamma(1-s)-s T-\ln |\mathcal{Y}|]
$$

where

$$
\Lambda(R, s)= \begin{cases}\gamma(s)-R, & s \geq s_{R} \\ s \gamma^{\prime}\left(s_{R}\right), & s<s_{R}\end{cases}
$$


$\gamma^{\prime}(s)=\frac{d \gamma(s)}{d s}$ and where $s_{R}$ is the solution to the equation

$$
\gamma(s)-s \gamma^{\prime}(s)=R,
$$

and similarly to (6)

$$
E_{2}^{*}(R, T)=E_{1}^{*}(R, T)+T .
$$

Let $\delta_{G V}(R)$ denote the normalized Gilbert-Varshamov (GV) distance, $\delta_{G V}=d_{G V} / n$, i.e., the smaller solution, $\delta$, to the equation

$$
h(\delta)=\ln (2)-R,
$$

where $h(\delta)=-\delta \ln (\delta)-(1-\delta) \ln (1-\delta)$ is the binary entropy function.

For the BSC with uniform ${ }^{1}$ random coding distribution, the upper bound is given by

$$
\begin{gathered}
\sup _{s \geq 0} E_{1}(R, T, s) \triangleq \\
\sup _{s \geq 0}\left(\mu(s, R)+s \ln \frac{1}{1-p}-\ln \left[p^{1-s}+(1-p)^{1-s}\right]-s T\right) \\
\mu(s, R)=\left\{\begin{array}{cc}
\mu_{0}(s, R) & s \geq s_{R} \\
\beta s \delta_{G V}(R) & s<s_{R}
\end{array}\right.
\end{gathered}
$$

where

$$
\mu_{0}(s, R)=s \ln (1-p)-\ln \left[p^{s}+(1-p)^{s}\right]+\ln 2-R
$$

and

$$
\beta=\ln \frac{1-p}{p} .
$$

It is noted that the optimal $s$ in (13) has an explicit expression given in [9]. .

\section{Main Results}

The main results in this paper are stated in Theorems 1 and 2 establishing exact expressions for the random coding error exponents $e_{1}(R, T)$ and $e_{2}(R, T)$ for the general DMC. Due to space limitation the proofs of all the results excluding Theorem 1 do are omitted and can be found in [16].

For a given probability distribution $Q$ on $\mathcal{X} \times \mathcal{Y}$ define

$$
\begin{aligned}
K(Q, R) & =\boldsymbol{E}_{Q} \ln \frac{1}{P(X)}-H_{Q}(X \mid Y)-R \\
& =D(Q(X) \| P(X))+I_{Q}(X ; Y)-R,
\end{aligned}
$$

and for a given probability distribution $Q_{Y}$ on $\mathcal{Y}$, define

$$
\mathcal{G}_{R}\left(Q_{Y}\right) \triangleq\left\{Q_{X \mid Y}: K(Q, R) \leq 0\right\},
$$

where $Q$ is the probability distribution $Q_{Y} \times Q_{X \mid Y}$.

Theorem 1 The error exponent $e_{1}(R, T)$ is given by

$$
e_{1}(R, T)=\min _{\tilde{Q}}\left[D(\tilde{Q} \| P(X, Y))+\min _{Q_{X \mid Y}} K(Q, R)\right],
$$

\footnotetext{
${ }^{1}$ By the term uniform random coding distribution we mean uniform over $\{0,1\}^{n}$
}

where $^{2} Q=\tilde{Q}_{Y} \times Q_{X \mid Y}$, the inner minimization is over $Q_{X \mid Y} \in \mathcal{G}_{R}^{c}\left(\tilde{Q}_{Y}\right)$ such that $\Omega(\tilde{Q}, Q, T) \leq 0, \tilde{Q}$ is a probability distribution on $\mathcal{X} \times \mathcal{Y}$, and

$$
\Omega(\tilde{Q}, Q, T) \triangleq \boldsymbol{E}_{\tilde{Q}} \ln P(Y \mid X)+\boldsymbol{E}_{Q} \ln \frac{1}{P(Y \mid X)}-T .
$$

Corollary 1 Under Condition 1 (see (7)) the error exponent $e_{1}(R, T)$ is given by

$$
\min _{\tilde{Q}: \xi(\tilde{Q}, T) \leq \gamma^{\prime}\left(s_{R}\right)}[D(\tilde{Q} \| P(X, Y))+\psi(s(\xi(\tilde{Q}, T)))]-R,
$$

where

$$
\xi(\tilde{Q}, T)=T+\boldsymbol{E}_{\tilde{Q}} \ln \frac{1}{P(Y \mid X)},
$$

$s(\xi)$ is the solution of the equation $\gamma^{\prime}(s)=\xi$, and

$$
\psi(s) \triangleq \gamma(s)-s \gamma^{\prime}(s) .
$$

Corollary 2 For the BSC with uniform random coding distribution, if $R \geq \ln 2-h(p+T / \beta), e_{1}(R, T)=0$ and otherwise

$$
\begin{aligned}
& e_{1}(R, T)= \\
& \min _{q \in\left[p, \delta_{G V}(R)-T / \beta\right]}[D(q \| p)-h(q+T / \beta)]+\ln 2-R,
\end{aligned}
$$

where $\beta$ is defined in (16).

It is easy to verify that the minimizing $q$ is either a boundary point of the interval $\left[p, \delta_{G V}(R)-T / \beta\right]$, or $q$ that satisfies the quadratic equation

$$
q^{2}\left(1-e^{-\beta}\right)+q\left(T / \beta\left(1-e^{-\beta}\right)+2 e^{-\beta}\right)-e^{-\beta}=0 .
$$

We note that in the BSC case the the exact exponent $e_{1}(R, T)$ (24) has an explicit expression and its simplicity relatively to the bounds $E_{1}(R, T)$ (4) and $E_{1}^{*}(R, T)$ in (13) is surprising.

Theorem 2 The error exponent $e_{2}(R, T)$ is given by

$$
\begin{array}{r}
e_{2}(R, T)=\min _{Q_{Y}}\left[D\left(Q_{Y} \| P_{Y}\right)+\min _{\Theta \leq \Theta_{0}\left(Q_{Y}\right)}\left\{E_{\mathcal{A}}\left(Q_{Y}, \Theta\right)\right.\right. \\
\left.\left.+E_{\mathcal{B}}\left(Q_{Y}, \Theta\right)\right\}-R\right],
\end{array}
$$

where

$\Theta_{0}\left(Q_{Y}\right)=\min _{Q_{X \mid Y} \in \mathcal{G}_{R}\left(Q_{Y}\right)}\left[\boldsymbol{E}_{Q} \ln \frac{1}{P(X, Y)}-H_{Q}(X \mid Y)\right]-R$, with $Q=Q_{Y} \times Q_{X \mid Y}$,

$$
\begin{aligned}
& E_{\mathcal{A}}\left(Q_{Y}, \Theta\right)= \\
& \min _{Q_{X \mid Y}: \boldsymbol{E}_{Q} \ln P(Y \mid X)=T-\Theta}\left[\boldsymbol{E}_{Q} \ln \frac{1}{P(X)}-H_{Q}(X \mid Y)\right], \\
& E_{\mathcal{B}}\left(Q_{Y}, \Theta\right)= \\
& \min _{Q_{X \mid Y}: \boldsymbol{E}_{Q} \ln P(Y \mid X) \leq-\Theta}\left[\boldsymbol{E}_{Q} \ln \frac{1}{P(X \mid Y)}-H_{Q}(X \mid Y)\right] .
\end{aligned}
$$

\footnotetext{
${ }^{2}$ that is, $Q$ and $\tilde{Q}$ share the same marginal pmf of $Y$.
} 
Corollary 3 For the BSC with uniform random coding distribution,

$$
e_{1}(R, T)=e_{2}(R, T)+T \text {. }
$$

The relation $e_{1}(R, T)=e_{2}(R, T)+T$, which is proved to hold in the case of the BSC with uniform random coding distribution, is not surprising. The intuition behind it can be explained as follows: Recall that the decision rule (2) was chosen to minimize the tradeoff between $\operatorname{Pr}\left\{\mathcal{E}_{1}\right\}$ and $\operatorname{Pr}\left\{\mathcal{E}_{2}\right\}$, and consider an equivalent problem of minimizing a Lagrangian which is a linear combination of $\operatorname{Pr}\left\{\mathcal{E}_{1}\right\}$ and $\operatorname{Pr}\left\{\mathcal{E}_{2}\right\}$, where the Lagrange multiplier is $e^{n T}$. Had $e_{1}(R, T)$ been different from $e_{2}(R, T)+T$, the exponents of these two terms would differ, and hence one could improve the overall exponent by changing their balance.

While our results imply that $e_{i}(R, T) \geq E_{i}(R, T)$ and $e_{i}(R, T) \geq E_{i}^{*}(R, T), i=1,2$, we have not been able to determine analytically whether or not there are cases in which at least one of these inequalities is a strong one. If such cases will be found, then the conclusion will be that we have strictly improved on the results of [1] or [9] or both. If not, then the conclusion would be that the exponents of [1] and [9] are tight, a fact which was not determined unequivocally before. In either case, the tools proposed in this paper provide us with a yardstick to determine the tightness of the results in [1] and [9]. As mentioned earlier, we have conducted a numerical study for the case of the BSC with uniform random coding distribution which indicates that in this case, $e_{i}(R, T)$ appearing in (24) is equal to $E_{i}^{*}(R, T)$ (see (8)) and to $E_{i}(R, T)$. We have shown analytically for this case that the lowest rate for which $e_{1}(R, T)=0$ is equal to that of $E_{1}^{*}(R, T)$.

\section{Derivation of The EXPONENT $e_{1}(R, T)$}

The probability of error given that the message that was sent is $m$, averaged over the codebooks is given by

$$
\begin{aligned}
\overline{\operatorname{Pr}}\left(\mathcal{E}_{1} \mid m\right)= & \sum_{\boldsymbol{x}_{m}} P\left(\boldsymbol{x}_{m}\right) \sum_{\boldsymbol{y}} P\left(\boldsymbol{y} \mid \boldsymbol{x}_{m}\right) \\
& \cdot \operatorname{Pr}\left\{\sum_{m^{\prime} \neq m} P\left(\boldsymbol{y} \mid \boldsymbol{x}_{m^{\prime}}\right)>P\left(\boldsymbol{y} \mid \boldsymbol{x}_{m}\right) e^{-n T} \mid \boldsymbol{x}_{m}, \boldsymbol{y}\right\} .
\end{aligned}
$$

Next, let $Q$ be an empirical probability distribution defined on $\mathcal{Y} \times \mathcal{X}$ and let $N_{\boldsymbol{y}}(Q)$ denote the number of codewords (excluding $\boldsymbol{x}_{m}$ ) whose joint empirical probability distribution with $\boldsymbol{y}$ is $Q$, and denote $f(\boldsymbol{y}, Q) \triangleq N_{\boldsymbol{y}}(Q) e^{n \boldsymbol{E}_{Q} \ln P(Y \mid X)}$ then

$$
\begin{aligned}
& \operatorname{Pr}\left\{\sum_{m^{\prime} \neq m} P\left(\boldsymbol{y} \mid \boldsymbol{x}_{m^{\prime}}\right)>P\left(\boldsymbol{y} \mid \boldsymbol{x}_{m}\right) e^{-n T} \mid \boldsymbol{x}_{m}, \boldsymbol{y}\right\} \\
& =\operatorname{Pr}\left\{\sum_{Q} N_{\boldsymbol{y}}(Q) e^{n \boldsymbol{E}_{Q} \ln P(Y \mid X)}>P\left(\boldsymbol{y} \mid \boldsymbol{x}_{m}\right) e^{-n T} \mid \boldsymbol{x}_{m}, \boldsymbol{y}\right\}
\end{aligned}
$$

$$
\begin{aligned}
& =\operatorname{Pr}\left\{\sum_{Q} f(\boldsymbol{y}, Q)>P\left(\boldsymbol{y} \mid \boldsymbol{x}_{m}\right) e^{-n T} \mid \boldsymbol{x}_{m}, \boldsymbol{y}\right\} \\
& \stackrel{(a)}{\doteq} \operatorname{Pr}\left\{\max _{Q} f(\boldsymbol{y}, Q)>P\left(\boldsymbol{y} \mid \boldsymbol{x}_{m}\right) e^{-n T} \mid \boldsymbol{x}_{m}, \boldsymbol{y}\right\} \\
& =\operatorname{Pr}\left\{\bigcup_{Q}\left\{f(\boldsymbol{y}, Q)>P\left(\boldsymbol{y} \mid \boldsymbol{x}_{m}\right) e^{-n T}\right\} \mid \boldsymbol{x}_{m}, \boldsymbol{y}\right\} \\
& \doteq \sum_{Q} \operatorname{Pr}\left\{f(\boldsymbol{y}, Q)>P\left(\boldsymbol{y} \mid \boldsymbol{x}_{m}\right) e^{-n T} \mid \boldsymbol{x}_{m}, \boldsymbol{y}\right\} \\
& \doteq \max _{Q} \operatorname{Pr}\left\{N \boldsymbol{y}(Q)>P\left(\boldsymbol{y} \mid \boldsymbol{x}_{m}\right) e^{n\left[\boldsymbol{E}_{Q} \ln \frac{1}{P(Y \mid X)}-T\right]} \mid \boldsymbol{x}_{m}, \boldsymbol{y}\right\},
\end{aligned}
$$

where $(a)$ follows from monotonicity and continuity of the exponent $^{3}$ in $T$. Now, we calculate

$$
\operatorname{Pr}\left\{N \boldsymbol{y}(Q)>P\left(\boldsymbol{y} \mid \boldsymbol{x}_{m}\right) e^{n\left[\boldsymbol{E}_{Q} \ln \frac{1}{P(Y \mid X)}-T\right]}\right\} .
$$

Recall the definition of $\Omega(\tilde{Q}, Q, T)$ (20), and note that

$$
\begin{aligned}
& \frac{1}{n} \ln \left(P\left(\boldsymbol{y} \mid \boldsymbol{x}_{m}\right) e^{n\left[\boldsymbol{E}_{Q} \ln \frac{1}{P(Y \mid X)}-T\right]}\right) \\
= & \boldsymbol{E}_{\hat{P}} \boldsymbol{x}_{m}, \boldsymbol{y} \ln P(Y \mid X)+\boldsymbol{E}_{Q} \ln \frac{1}{P(Y \mid X)}-T \\
= & \Omega\left(\hat{P}_{\boldsymbol{x}_{m}, \boldsymbol{y}}, Q, T\right),
\end{aligned}
$$

so we will be interested in evaluating $\operatorname{Pr}\left\{N_{\boldsymbol{y}}(Q)>e^{n \Omega\left(\hat{P}_{\boldsymbol{x}}, \boldsymbol{y}, Q, T\right)}\right\}$. There are two cases to consider depending on the sign of $\Omega=\Omega\left(\hat{P}_{\boldsymbol{x}_{m}, \boldsymbol{y}}, Q, T\right)$.

The case $\Omega \leq 0$ : Here $e^{n \Omega} \leq 1$ and since $N_{\boldsymbol{y}}(Q)$ takes on integer values,

$$
\begin{aligned}
& \operatorname{Pr}\left\{N_{\boldsymbol{y}}(Q)>e^{n \Omega}\right\} \\
& =\operatorname{Pr}\left\{N_{\boldsymbol{y}}(Q) \geq 1\right\} \\
& \doteq 1-\left[1-e^{n\left[H_{Q}(X \mid Y)+\boldsymbol{E}_{Q} \ln P(X)\right]}\right]^{M-1} \\
& \doteq \exp \left\{-n\left|-H_{Q}(X \mid Y)-\boldsymbol{E}_{Q} \ln P(X)-R\right|^{+}\right\} \\
& =\exp \left\{-n|K(Q, R)|^{+}\right\},
\end{aligned}
$$

where $K(Q, R)$ is defined in (17), the first exponential equality is because the random variable $N_{\boldsymbol{y}}(Q)$ is equal to the sum of the i.i.d. binary- $p$ random variables $\mathbf{1}\left\{\hat{P}_{\boldsymbol{X}_{i}, \boldsymbol{y}}=Q\right\}, i=$ $1, \ldots, e^{n R}-1$, with $p \doteq e^{n\left[H_{Q}(X \mid Y)+\boldsymbol{E}_{Q} \ln P(X)\right]}$, and the second exponential equality is because if $a \in[0,1]$, then $\frac{1}{2} \min \{1, a M\} \leq 1-(1-a)^{M} \leq \min \{1, a M\}$ (see Lemma 1 in [15]).

The case $\Omega>0$ : There are two sub-cases to consider:

${ }^{3}$ Formally, the difference between $\sum_{Q} f(\boldsymbol{y}, Q)$ and $\max _{Q} f(\boldsymbol{y}, Q)$, which is $O(\log n / n)$ in the exponential scale, can be absorbed in the parameter $T$. Thus, one can derive upper and lower bounds in terms of $e_{1}(R, T+$ $O(\log n / n))$ and $e_{1}(R, T-O(\log n / n))$, with $e_{1}(\cdot, \cdot)$ being defined as in (19). These are asymptotically the same wherever $e_{1}(R, T)$ is continuous in $T$. 
- If $\Omega>0$ and $\Omega \geq-K(Q, R)$ we can use the Chernoff bound, similarly to [10] Appendix B

$$
\begin{aligned}
& \operatorname{Pr}\left\{N_{\boldsymbol{y}}(Q)>e^{n \Omega}\right\} \\
& \leq \min \left\{1, \exp \left\{-e^{n \Omega}[n\{K(Q, R)+\Omega\}-1]\right\}\right\} .
\end{aligned}
$$

This term decays at least double-exponentially and is negligible in the exponential scale.

- If $0<\Omega \leq-K(Q, R)$ we prove again, using Chernoff bound similarly to [10] Appendix B that $\operatorname{Pr}\left\{N_{\boldsymbol{y}}(Q)>e^{n \Omega}\right\}$ is very close to one.

Therefore, for $\Omega>0$, we have

$$
\operatorname{Pr}\left\{N_{\boldsymbol{y}}(Q)>e^{n \Omega}\right\} \doteq \mathbf{1}\{0<\Omega \leq-K(Q, R)\}
$$

Combining this with the expression for the range where $\Omega \leq 0$ (32), we get.

$$
\begin{aligned}
\operatorname{Pr}\left\{N_{\boldsymbol{y}}(Q)>e^{n \Omega}\right\} \doteq & \mathbf{1}\{\Omega \leq 0\} \exp \left\{-n|K(Q, R)|^{+}\right\} \\
& +\mathbf{1}\{0<\Omega \leq-K(Q, R)\} .
\end{aligned}
$$

This can be rewritten as

$$
\begin{aligned}
\operatorname{Pr} & \left\{N_{\boldsymbol{y}}(Q)>e^{n \Omega}\right\} \\
\doteq & \mathbf{1}\left\{Q \in \mathcal{G}_{R}^{c}\left(\hat{P}_{\boldsymbol{y}}\right), \Omega \leq 0\right\} \exp \{-n(K(Q, R))\} \\
& +\mathbf{1}\left\{Q \in \mathcal{G}_{R}\left(\hat{P}_{\boldsymbol{y}}\right), \Omega \leq-K(Q, R)\right\} .
\end{aligned}
$$

Recall that, in fact, $\Omega=\Omega(\tilde{Q}, Q, T)$ (with $\tilde{Q}=\hat{P}_{\boldsymbol{x}_{m}, \boldsymbol{y}}$, see (31)), so the condition in the second term, $\Omega \leq-K(Q, R)=$ $H_{Q}(X \mid Y)+\boldsymbol{E}_{Q} \ln P(X)+R$ is equivalent to $E_{\tilde{Q}} \ln P(Y \mid X)-$ $T \leq R+\boldsymbol{E}_{Q} \ln P(X, Y)+H_{Q}(X \mid Y)$.

Thus, after maximizing over $Q$ and taking the expectation w.r.t. $\left(\boldsymbol{x}_{m}, \boldsymbol{y}\right)$, the resulting exponent is

$$
e_{1}(R, T)=\min \left\{E_{a}(R, T), E_{b}(R, T)\right\}
$$

where

$$
\begin{aligned}
E_{a}(R, T)= & \min _{\tilde{Q}}[D(\tilde{Q} \| P(X, Y)) \\
& \left.+\min _{Q_{X \mid Y}: Q \in \mathcal{G}_{R}^{c}\left(\tilde{Q}_{Y}\right), \Omega(\tilde{Q}, Q, T) \leq 0} K(Q, R)\right], \\
E_{b}(R, T)= & \min _{\tilde{Q} \in \mathcal{L}_{R, T}} D(\tilde{Q} \| P(X, Y)),
\end{aligned}
$$

with $Q=\tilde{Q}_{Y} \times Q_{X \mid Y}$, and where

$\mathcal{L}_{R, T}=\left\{\begin{array}{l}\tilde{Q}(X, Y): E_{\tilde{Q}} \ln P(Y \mid X) \leq R+T+ \\ \max _{Q \in \mathcal{G}_{R}\left(\tilde{Q}_{Y}\right)}\left[\boldsymbol{E}_{Q} \ln P(X, Y)+H_{Q}(X \mid Y)\right]\end{array}\right\}$,

and the probability distributions $\tilde{Q}$ are defined on the set $\mathcal{X} \times$ $\mathcal{Y}$. Next, we show that $E_{a}(R, T)$ is the dominant term in the minimization (37)

$$
\begin{aligned}
& E_{a}(R, T)= \\
& \min _{\tilde{Q}}\left[D(\tilde{Q} \| P(X, Y))+\min _{Q \in \mathcal{G}_{R}^{c}\left(\tilde{Q}_{Y}\right): \Omega(\tilde{Q}, Q, T) \leq 0} K(Q, R)\right]
\end{aligned}
$$

$$
\begin{aligned}
& \leq \min _{\tilde{Q} \in \mathcal{L}_{R, T}}\left[D(\tilde{Q} \| P(X, Y))+\min _{Q \in \mathcal{G}_{R}^{c}\left(\tilde{Q}_{Y}\right): \Omega(\tilde{Q}, Q, T) \leq 0} K(Q, R)\right] \\
& \stackrel{(a)}{=} \min _{\tilde{Q} \in \mathcal{L}_{R, T}}\left[D(\tilde{Q} \| P(X, Y))+\min _{Q \in \mathcal{G}_{R}^{c}} K(Q, R)\right] \\
& \stackrel{(b)}{=} \min _{\tilde{Q} \in \mathcal{L}_{R, T}} D(\tilde{Q} \| P(X, Y)) \\
& =E_{b}(R, T),
\end{aligned}
$$

where $(a)$ follows since when $\tilde{Q} \in \mathcal{L}_{R, T}$, the constraint $\Omega(\tilde{Q}, Q, T) \leq 0$ becomes inactive, and $(b)$ is by definition of $\mathcal{G}_{R}\left(\tilde{Q}_{Y}\right)$, and by the fact that when the rate $R$ is below capacity, the boundary of $\mathcal{G}_{R}^{c}\left(\tilde{Q}_{Y}\right)$ is non empty .

This results in the exponent $e_{1}(R, T)=E_{a}(R, T)$.

\section{REFERENCES}

[1] G. D. Forney, Jr, "Exponential error bounds for erasure, list, and decision feedback schemes," IEEE Trans. Inform. Theory, vol. IT-14, no. 2, pp. 206-220, Mar. 1968.

[2] R. Ahlswede, N. Cai, and Z. Zhang, "Erasure, list, and detection zeroerror capacities for low noise and a relation to identication," IEEE Trans. Inform. Theory, vol. 42, no. 1, pp. 55-62, Jan. 1996.

[3] T. Hashimoto, Composite scheme LR+Th for decoding with era- sures and its effective equivalence to Forneys rule, IEEE Trans. Inform. Theory, vol. 45, no. 1, pp. 78-93, Jan. 1999.

[4] T. Hashimoto and M. Taguchi, Performance and explicit error detec- tion and threshold decision in decoding with erasures, IEEE Trans. Inform. Theory, vol. 43, no. 5, pp. 1650-1655, Sep. 1997.

[5] P. Kumar, Y.-H. Nam, and H. El Gamal, On the error exponents of arq channels with deadlines, IEEE Trans. Inform. Theory, vol. 53, no. 11, pp. 4265-4273, Nov. 2007.

[6] N. Merhav and M. Feder, Minimax universal decoding with an erasure option, IEEE Trans. Inform. Theory, vol. 53, no. 5, pp. 1664-1675, May 2007.

[7] A. J. Viterbi, Error bounds for the white Gaussian and other very noisy memoryless channels with generalized decision regions, IEEE Trans. Inform. Theory, vol. IT-15, no. 2, pp. 279-287, Mar. 1969.

[8] M. Mézard and A. Montanari, Information, Physics, and Computation, Oxford University Press, 2009.

[9] N. Merhav, "Error exponents of erasure/list decoding revisited via moments of distance enumerators," IEEE Trans. Inform. Theory, vol. 54, no. 10, pp. 4439-4447, Oct. 2008.

[10] N. Merhav, "Relations between random coding exponents and the statistical physics of random codes," IEEE Trans. Inform. Theory, vol. 55, no. 1, pp. 83-92, Jan. 2009.

[11] N. Merhav, "The generalized random energy model and its application to the statistical physics of ensembles of hierarchical codes," IEEE Trans. Inform. Theory, vol. 55, no. 3, pp. 1250-1268, Mar. 2009.

[12] R. Etkin, N. Merhav and E. Ordentlich, "Error exponents of optimum decoding for the interference channel," IEEE Trans. Inform. Theory, vol. 56 no. 1 pp. 40-56, Jan. 2010.

[13] Y. Kaspi and N. Merhav, "Error exponents for broadcast channels with degraded message sets," submitted to IEEE Trans. Inform. Theory, May 2009.

[14] R. G. Gallager, Information Theory and Reliable Communication. New York: Wiley, 1968.

[15] A. Somekh-Baruch and N. Merhav, "Achievable error exponents for the private fingerprinting game," vol. 53, no. 5, pp. 1827-1838, May 2007.

[16] A. Somekh-Baruch and N. Merhav, "Exact random coding exponents for erasure decoding," in preparation, Jan. 2010.

\footnotetext{
${ }^{4}$ To realize this, note that for rates below the capacity $P \in \mathcal{G}_{R}^{c}\left(\tilde{Q}_{Y}\right)$ since $\left.\left[-H_{Q}(X \mid Y)-\boldsymbol{E}_{Q} \ln P(X)\right]\right|_{Q=P}-R=I_{P}(X ; Y)-R \leq 0$, and obviously $\mathcal{G}_{R}\left(\tilde{Q}_{Y}\right)$ is not empty, e.g. it contains $Q_{X, Y}=P(X) Q_{Y}$ which yields $\left[-H_{Q}(X \mid Y)-\boldsymbol{E}_{Q} \ln P(X)\right]-R=-R$ hence, and by continuity there exists a linear combination of these two probability distributions that lies on the boundary of $\mathcal{G}_{R}^{c}\left(\tilde{Q}_{Y}\right)$.
} 